\title{
A Bayesian approach to person perception
}

Clifford, C.W.G. ${ }^{1}$, Mareschal, I. ${ }^{2}$, Otsuka, Y. ${ }^{1} \&$ Watson, T.L. ${ }^{3}$

1. School of Psychology, UNSW Australia, Sydney, New South Wales, Australia.

2. School of Biological and Chemical Sciences, Psychology, Queen Mary University of London, London, UK.

3. School of Social Sciences and Psychology, The University of Western Sydney, Bankstown, New South Wales, Australia.

Corresponding author:

\section{Colin Clifford}

E: colin.clifford@unsw.edu.au

$\mathrm{T}:+61-(0) 2-9385-1050$

$\mathrm{F}:+61-(0) 2-9385-3641$ 


\section{Abstract}

Here we propose a Bayesian approach to person perception, outlining the theoretical position and a methodological framework for testing the predictions experimentally. We use the term person perception to refer not only to the perception of others' personal attributes such as age and sex but also to the perception of social signals such as direction of gaze and emotional expression. The Bayesian approach provides a formal description of the way in which our perception combines current sensory evidence with prior expectations about the structure of the environment. Such expectations can lead to unconscious biases in our perception that are particularly evident when sensory evidence is uncertain. We illustrate the ideas with reference to our recent studies on gaze perception which show that people have a bias to perceive the gaze of others as directed towards themselves. We also describe a potential application to the study of the perception of a person's sex, in which a bias towards perceiving males is typically observed.

\section{Introduction}

When we look at another person what do we expect to see? And what role do those expectations play in the processing of our sensory input and in determining what we perceive? When we look at a body or a face we have a clear expectation of its basic structure in terms of the constituent elements and their configuration (Figure 1). There are also well-documented asymmetries or biases in the perception of attributes such as sex and age indicating a role for prior expectation at a perceptual and/or cognitive level. For example, when presented with an image of a face, there is a bias to respond 'male' when asked about that person's sex (e.g. Armann \& Bulthoff, 2012) and to judge their age as closer to your own (e.g. Voelkle et al., 2012). However, there is currently no adequate theoretical framework within which to interpret these effects or to generate new testable predictions. This leaves the field with no clear idea about how perceptual and decision processes are dissociated and therefore at what level prior expectations are operating and biases generated. Specifically, are these perceptual biases in the processing of sensory information that actually affect the way things look to the observer or are they cognitive response biases in the decision criteria used to categorize that sensory information? This is an important question because such biases may have a large impact on how we interact with people in a range of consequential settings.

To establish the level at which prior expectations are implemented in the system, we propose a Bayesian framework for the study of person perception. We do not intend the term "person perception" to be taken to refer to the perception of identity, although we speculate that the Bayesian framework may also be of relevance to identity recognition in the sense that information about the context in which a person is encountered provides prior expectation as to who they are. The Bayesian framework provides a principled approach to understanding person perception using a method that has proven successful in designing and interpreting psychophysical experiments in the sensory and motor domains (see Kersten et al., 2004; Knill \& Pouget, 2004; Trommershäuser et al., 2011). 


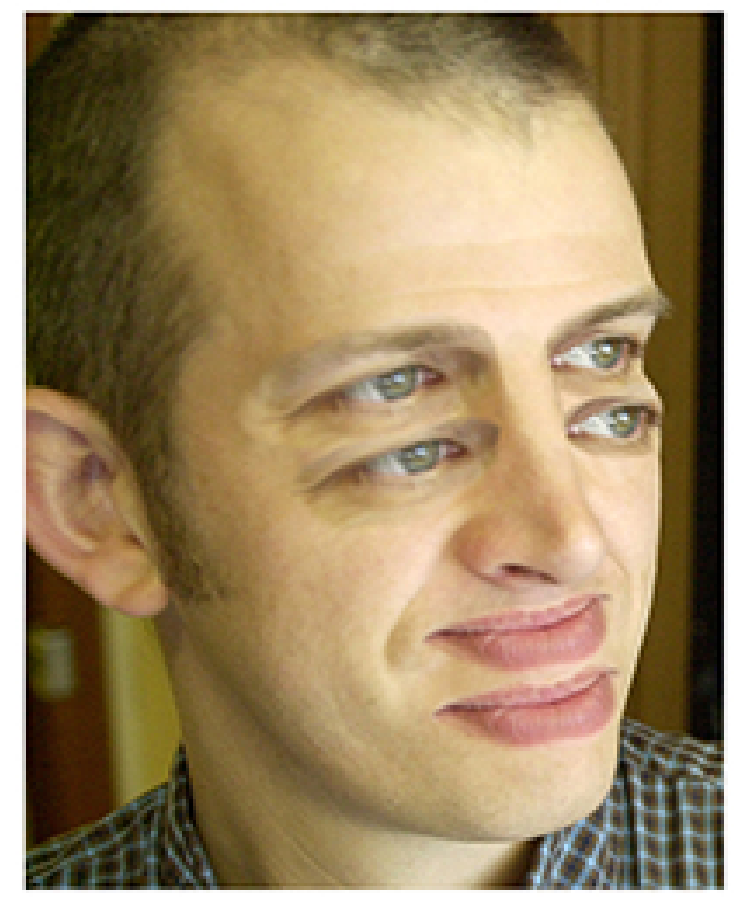

A Bayesian estimator combines sensory evidence with stored knowledge, represented as the prior probability distribution. The prior probability distribution can embody not only expectations about the structure of the environment (e.g. Stocker \& Simoncelli, 2006a; Girshick et al., 2011) but also the likely consequences of errors for the observer (Haselton \& Buss, 2000; Haselton \& Nettle, 2006). For example, we expect the structure of a face to be two eyes above a noise and a mouth (and can feel quite disconcerted when that expectation is violated, as in Figure 1). In terms of errors, the consequence of misidentifying a man as a woman might be more costly than misidentifying a woman as a man and the cost of missing an instance of gaze directed at you might be greater than the cost of a false alarm when gaze is in fact directed elsewhere.

The Bayesian approach has proved influential in understanding the role of prior expectation in vision. For example, experimental evidence indicates that humans have a prior expectation for motion to be slow (Stocker \& Simoncelli, 2006a), contours to be horizontal or vertical (Girshick et al., 2011) and lighting to come from above and slightly to the left (Gerardin et al., 2010). A Bayesian framework has also been proposed in the context of social cognition and its disorders in conditions such as autism and schizophrenia (Kilner et al., 2007; Fletcher \& Frith, 2009; Hohwy \& Palmer, 2014). In a recent paper, we applied the Bayesian approach for the first time to the processing of a socially relevant perceptual feature, namely the direction of another's gaze (Mareschal et al., 2013b). This has provided an important insight into gaze perception, namely that humans have an expectation that gaze is directed towards them that acts to "pull" the perceived direction of gaze towards the observer, particularly under conditions of high stimulus uncertainty (see also Mareschal et al., 2014; Martin \& Jones, 1982; Martin \& Rovira, 1981; Sheldrake, 2003). We believe that this approach will prove readily extendable to the investigation of how our visual systems deal with the task of extracting socially relevant information from often uncertain, incomplete or ambiguous visual input to the perception of personal attributes from images of the face and body. 


\section{Bias in the Perception of a Person's Sex}

As an illustration of the potential applicability of the Bayesian approach, consider the perception of a person's sex. There is a well-established bias to respond 'male' when asked to report a person's sex from an image of their face (e.g. Cellerino et al, 2004; Armann \& Bulthoff, 2012) that has also been reported in chimpanzees (de Waal \& Pokorny, 2008). A similar 'male bias' has also been found for body shape (Johnson et al, 2012), hands (Gaetano et al, 2014) and biological motion (Troje et al, 2006). It has been suggested that, in the history of humans, misclassifying a man as female has generally proved to be potentially more dangerous than misclassifying a woman as male (Armann \& Bulthoff, 2012). A similar "better safe than sorry" heuristic has also been invoked in the context of the bias for direct gaze (Mareschal et al., 2013b) and other social judgments (Johnson et al., 2012; Haselton \& Nettle, 2006). Whenever judgments are made under uncertainty and the costs of errors are asymmetrical in this way, it has been argued that human decision making should be biased toward making less costly errors. This bias might increase overall error rates, but it minimizes overall cost (Haselton \& Buss, 2000; Haselton \& Nettle, 2006).

A distinction has been made in the sex perception literature between perceptual biases in the processing of sensory information and cognitive response biases in the criteria used to categorize that information (e.g. Webster et al., 2004; Armann \& Bulthoff, 2012). A heuristic bias underlying our judgments about another person's sex could in principle be implemented at either (or both) of these levels. Through a Bayesian-inspired experimental approach, perceptual biases can be isolated experimentally by avoiding the use of a categorization judgment. This can be achieved by requiring subjects to make a forced-choice discrimination as to which of two stimuli appears more male (or more female) instead of reporting whether a particular stimulus appears male or female. Making a relative judgment between two stimuli allows us to avoid the issue of categorization and better isolate biases at the perceptual level. We can then model perception as a process of Bayesian estimation according to which sensory evidence is combined with stored knowledge, represented as the prior probability distribution.

\section{The Bayesian Framework: Sensory Representations, Likelihood Functions, Priors \& Posteriors}

On any given presentation of a stimulus attribute of value $\theta_{0}$, the observer's sensory representation of the stimulus provides a noisy estimate, $\theta_{N}$, of the value of that attribute. For the sake of analytic tractability we assume that, across trials, the noise in the sensory estimate can be approximated by a Gaussian distribution of mean zero and standard deviation $\sigma_{0}$. Hence, on any given presentation of a stimulus attribute of value $\theta_{0}$, the sensory estimate is drawn from a Gaussian probability distribution with mean $\theta_{0}$ and standard deviation $\sigma_{0}$. Under a Bayesian framework, this purely sensory estimate is combined with prior knowledge about the probable value of the attribute to produce a more robust estimate: $p\left(\theta \mid \theta_{N}\right) \propto L\left(\theta_{N} \mid \theta\right) p(\theta)$. In the context of, say, gaze perception, this means that the posterior probability distribution, $p\left(\theta \mid \theta_{N}\right)$, of the direction of gaze given the sensory representation is proportional to the likelihood of that representation given the stimulus, $L\left(\theta_{N} \mid \theta\right)$ multiplied by the prior probability of the direction of gaze, $p(\theta)$. If the noise, $\sigma_{0}$, in the sensory estimate is independent of the precise direction of gaze within the vicinity of $\theta_{0}$, then the likelihood function will also be Gaussian with standard deviation $\sigma_{0}$ (Figure 2, Left). Assuming that the prior probability distribution can also be described by a Gaussian, the posterior distribution for any given 
observation of gaze direction is itself Gaussian (Figure 2, Right), which greatly simplifies derivation of further analytic results.

A

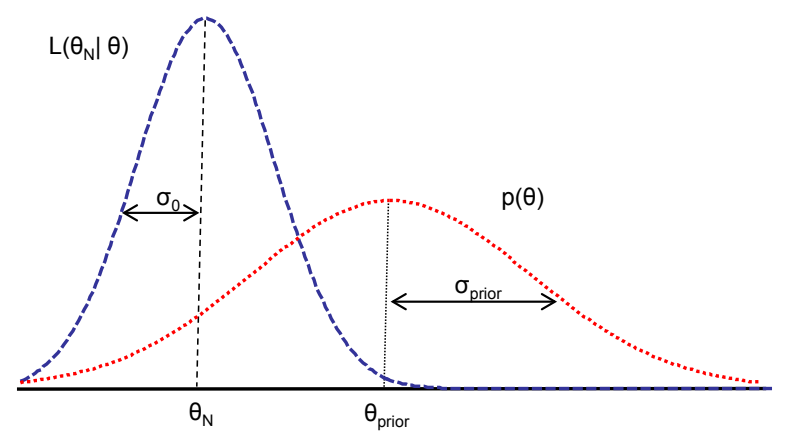

B

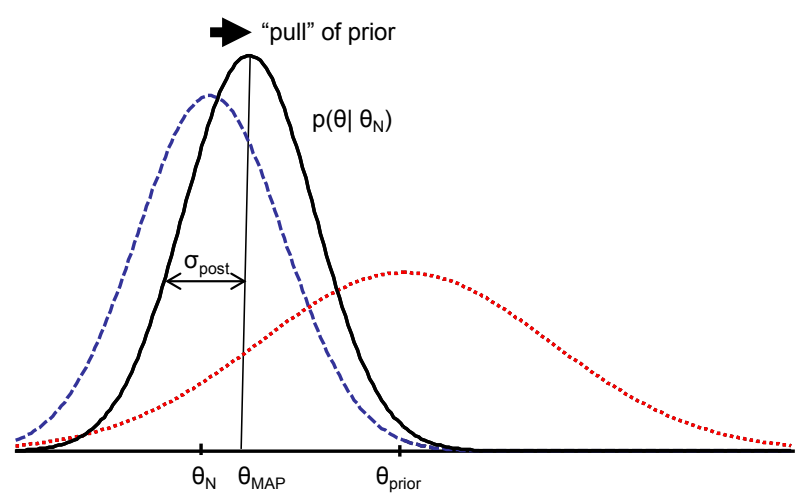

\section{A Psychophysical Task to Estimate the Prior Illustrated with Gaze Perception}

In recent studies we have taken a Bayesian experimental approach to investigate the role of prior expectation in the perception of others' direction of gaze, specifically whether someone is looking directly at us or to our left or right (Mareschal et al., 2013b, 2014). Gaze processing has the potential to serve as a useful model system for social neuroscience by virtue of its relative simplicity and the identification of neural mechanisms in the human and non-human primate brains (e.g. Perrett et al., 1985; Calder et al., 2007). Key to our experimental approach is the manipulation of stimulus uncertainty to recover the prior probability distribution that represents our stored knowledge. Within a Bayesian framework, increasing the uncertainty in a stimulus increases the influence of the prior, effectively "pulling" the resulting percept (taken to be the maximum a posteriori or MAP estimate of the attibute value) towards the peak of the prior distribution (Figure 2).

To quantify the effect of stimulus uncertainty on perception, we measure bias in the perception of specific stimulus attributes (e.g. horizontal gaze deviation) in a forced-choice task between stimuli differing in their uncertainty (e.g. Stocker \& Simoncelli, 2006a; Girshick et al., 2011; Mareschal et al., 2013b). Specifically, in our psychophysical task we present two stimuli at different levels of uncertainty, $\sigma_{1}$ and $\sigma_{2}$, associated with their respective sensory estimates. Subjects are asked to report which stimulus appears to have the attribute with the greater (or lesser) value (e.g. which face is gazing more to the left). To manipulate the level of uncertainty in the sensory representation we add noise to the eyes of the stimulus in the form of a pseudorandom texture while at the same 
time decreasing the contrast between the pupil and the sclera (Mareschal et al., 2013ab, 2014), as illustrated in Figure 3A.

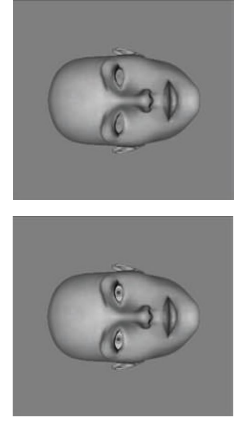

$\varangle$

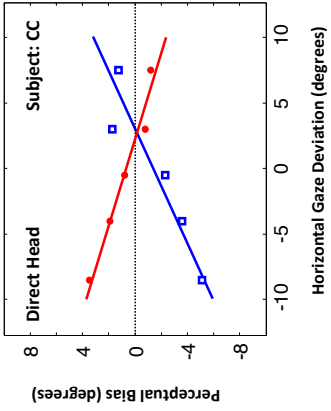

$\infty$

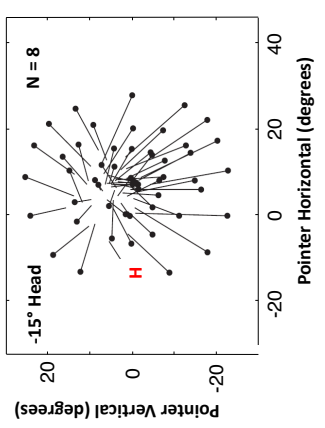

$u$

On the basis of the Bayesian framework outlined above, we can make the following qualitative predictions about the form of the experimental data: (1) the pull of the prior will be zero when the stimulus lies at the peak of the prior; (2) the direction of the pull will depend upon which side of the peak of the prior the stimulus lies; (3) the magnitude of the pull will increase with the uncertainty in the sensory representation of the stimulus; (4) for a Gaussian prior, the magnitude of the pull will increase linearly with the difference between the stimulus and the peak of the prior (Mareschal et al., 2013b). Together, these aspects of the Bayesian model predict the characteristic cross-shape of the data in Figure 3B where the $x$-position of the cross approximates the value of prior. The slope of the lines in Figure 3B depends on the uncertainty in the sensory representations of the stimuli, which can be estimated from discrimination data (as in Mareschal et al, 2013b) to produce a quantitative description of the prior.

To ensure that our findings generalize beyond the particular forced-choice task employed, and to directions of gaze deviation beyond horizontal, we developed an alternative task in which participants indicated the direction of gaze of a single stimulus by means of an on-screen pointer which they controlled via a computer mouse (Mareschal et al., 2014). Figure $3 C$ shows mean results from a cohort of 8 participants. Lines join data points from corresponding stimuli presented in noiseless (solid symbol) and noisy conditions. The resulting needles can be seen to point towards a direction close to direct gaze, consistent with the prior for direct gaze inferred from the data collected by Mareschal et al. (2013b) using the forced-choice task. This is the case not only for horizontal gaze deviations but for all gaze directions tested. It should be noted that the perceptual bias is towards direct gaze per se rather than towards the orientation of the head, as also observed by Mareschal et al. (2013b) using the forced-choice task, as head orientation is 15 degrees leftwards of direct, indicated by the position of the $\mathrm{H}$ in Figure $3 \mathrm{C}$. 
Evidence of a prior expectation for others' gaze to be directed towards us thus appears to generalize across head orientation, direction of gaze deviation, and behavioural task (Mareschal et al. (2013b, 2014). Future work might profitably employ an alternative operationalization of stimulus uncertainty, such as manipulation of stimulus duration, to confirm that this pattern of results does not depend upon the addition of noise to and reduction of contrast within the eyes.

\section{Structure of the Representational Space for Person Perception: Priors and Norms}

In the sensory coding literature it is common to talk about the representation of low-level perceptual attributes (e.g. speed of image motion) in terms of channels selective for a range of values of that attribute (e.g. fast and slow speed channels: Edwards et al., 1998). Previous work applying psychophysical adaptation to high-level vision has been used to make inferences about the way representations of the various attributes of faces are structured. The dimensions of this representational 'face space' include sex, identity, attractiveness, age, race and emotion as well as direction of gaze (e.g. Calder et al., 2008; Leopold et al., 2001; O'Neil \& Webster, 2011; Rhodes et al., 2003, 2004; Watson \& Clifford, 2003, 2006; Webster et al., 2004). The Bayesian approach is a natural complement to this work as it provides a direct way to recover the representational structure of this perceptual space. Our recent work on gaze perception (Mareschal et al, 2013b, 2014) provides the methodological and empirical foundations for the translation of the Bayesian approach from its successful application in low-level sensory and motor domains into high-level vision and person perception.

While the aim of the Bayesian approach is to elucidate the structure of long standing perceptual expectations about physical stimuli, psychophysical adaptation studies have been used to assess the underlying channel structure of face space (for a review see Webster and MacLeod, 2011). Adaptation studies suggest the perceptual face space assessed in these studies is centred on a neutral central point, or a norm. This is because adaptation to the end of a dimension in face space causes a negative aftereffect such that previously neutral faces take on the appearance of faces towards the opposite end of the continuum to the adaptor. For example, following adaptation to a series of male faces, a previously androgynous face will tend to appear female (Webster et al., 2004). The norm in this context can be seen either as the central tendency of the space as represented by a channel selective to that region or as a balance point between channels sensitive to opposing regions of the space (Webster and MacLeod, 2011; Rhodes et al., 2005).

The question, then, is how do the channel structure suggested by face adaptation studies and the prior suggested by the Bayesian approaches interact. The standard Bayesian population coding framework assumes that a perceptual variable is encoded in a set of noisy channel activations and that prior expectation is incorporated into a subsequent decoding stage that operates on the output of those channels (Zemel, Dayan \& Pouget, 1998). An intuitive hypothesis is that adaptation might change the prior distribution. Specifically, if the prior reflects the distribution of values of the perceptual variable in the world then repeated presentation of the same value should increase the prior probability in its vicinity. However, such a change in the prior distribution would lead to a perceptual bias that is opposite to the negative aftereffects typically observed perceptually (Stocker \& Simoncelli, 2006b; Clifford et al., 2007). 
A

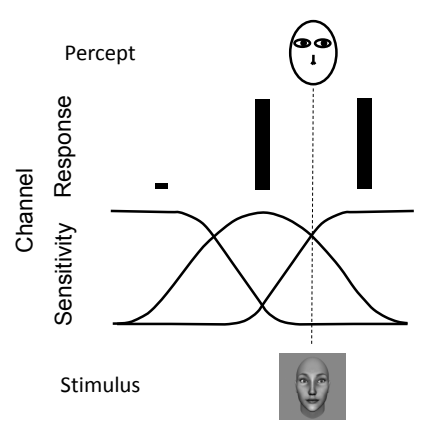

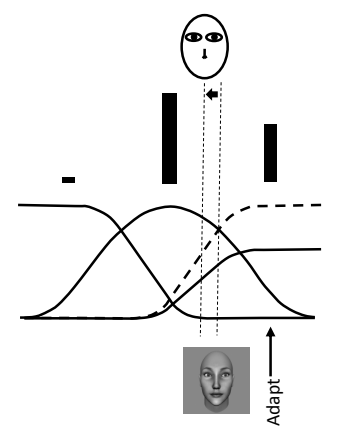

C

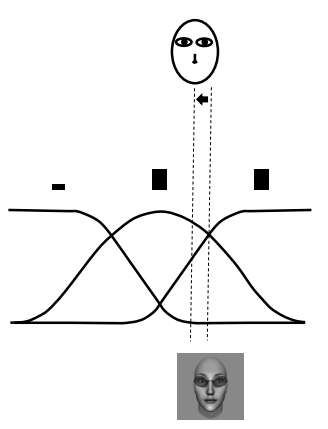

Alternatively, it has been proposed that prior expectation might be encoded implicitly within the channel structure itself rather than through an explicit decoding stage (Fischer \& Pena, 2011; Ganguli \& Simoncelli, 2014). Take, for example, the perception of gaze direction, where there is a prior towards perceiving direct gaze (Mareschal et al., 2013b, 2014). Adaptation studies indicate that gaze direction is coded by a multi-channel system involving channels representing various directions of gaze deviation (Jenkins et al., 2006; Cheleski et al., 2013) in addition to a channel explicitly representing direct gaze (Calder, Jenkins, Cassel and Clifford, 2008). A degree of baseline activity equal across these channels would tend to pull the representation of gaze direction in noisy stimuli towards their central tendency, namely direct gaze. If the level of this baseline activity was small compared to the channel activation generated by a stimulus of high certainty then the pull of the prior would diminish as stimulus certainty increased. Thus, in this instance of a multi-channel representation including an explicit channel for direct gaze, it appears that both the effects of perceptual adaptation and the prior could be instantiated through encoding within the same channel structure without the need to incorporate prior knowledge explicitly at the decoding stage (Figure 4). In this way, the effects of adaptation might be better understood in terms of short-term changes in channel sensitivity in response to an increase in signal-to-noise ratio of the sensory signal in the vicinity of the adaptor value (Wainwright, 1999), while the prior remains stable to reflect longterm environmental regularities.

This neat scenario, where both prior and channel structure are combined into a single model, need not apply to all decision categories. While adaptation is generally considered to uncover the structure of the encoded representation of the stimulus dimensions under investigation, the Bayesian approach could be measuring either an expectation that is encoded within the channel structure (Fischer \& Pena, 2011; Ganguli and Simoncelli, 2014) or one that becomes apparent not at the encoding but at a decoding stage (Zemel, Dayan \& Pouget, 1998). A full investigation of the form of any biases measured should uncover more about the relationship between the underlying representation proposed via adaptation and that proposed by a Bayesian approach.

The use of a Gaussian probability distribution to model perceptual prior expectation is motivated in large part by considerations of analytical tractability. However, the best description of a prior need 
not be Gaussian. For example, in domains of face perception where there are discrete categories, such as sex and race, priors might not be unimodal but instead contain multiple peaks each corresponding to the norm of a different category. Conceptualization of a perceptual norm as a peak within a prior distribution thus provides a natural way in which different dimensions of face space might be found experimentally to have one or many norms. To this end, we anticipate that mixture of Gaussian prior probability distributions (Knill, 2003) might provide a rich description of the expectations underlying our perception of faces.

\section{The Bayesian Approach as Unconscious Perceptual Inference}

The Bayesian approach as we put it forward imposes prior expectations on the sensory input and this could be achieved at perceptual levels of processing without requiring any cognitive inference. Even if the prior is considered to operate at the decoding stage of stimulus processing, rather than being implicit in the channel structure, this need not require any cognitive input. To uncover the prior for gaze direction, the studies outlined above (Mareschal et al., 2013b, 2014) used tasks designed to avoid the need for a categorical judgment. This minimized the possible contribution of cognitive biases, providing a result that can be incorporated into our understanding of how gaze direction is coded at a perceptual level. Indeed, one strength of the Bayesian approach is the potential to extend the experimental paradigm to manipulate the possibility of the participant introducing a cognitive bias into their decision making strategy. Teasing apart a perceptual from a cognitive bias in this way would provide a rich understanding of how processing of a stimulus can be biased at the most basic levels of visual processing and how this may interact with response criteria and other such decision processes at a more cognitive level.

\section{What does the Bayesian Approach to Person Perception Offer?}

By establishing the role of prior knowledge in person perception, we hope to achieve a better understanding of the way in which expectation shapes our processing of the social world. In addition to biases in the domains of gaze and sex perception, there are established biases in age estimation and attractiveness judgments. Experiments within a Bayesian framework have the potential to contribute to our understanding of each of these important aspects of person perception by making such biases explicable in a way that has not been possible before.

It has recently been suggested that the deficits in person perception associated with autism might be understood in a Bayesian framework in terms of weak or inefficient priors (Pellicano \& Burr, 2012). This may similarly apply to people with schizophrenia who also show changed person perception abilities (Watson, 2013). Thus, we anticipate that experiments conducted on samples of the normal population will likely seed further research with clinical populations in the near future.

We acknowledge that the Bayesian approach to perception tends to polarise researchers in the field and that there are situations where it can struggle to provide a parsimonious account of behavioural data (e.g. Anderson et al., 2011). However, the application of a Bayesian framework to person perception is a novel step that we believe will advance the field for the following reasons. Firstly, it provides an explicit computational approach generating empirically testable predictions. Secondly, it motivates a new class of experiments investigating the effect of uncertainty whose results will serve to constrain models (Bayesian or otherwise) of person perception. Thirdly, it will provoke discussion 
of the kinds of model that are appropriate for the perception of socially relevant stimuli and other aspects of social behaviour and what sort of evidence is required to choose between them.

\section{Acknowledgments:}

This work is supported by Australian Research Council Discovery Project DP120102589. CC is supported by Australian Research Council Future Fellowship FT110100150. 
Figure Legends:

Figure 1. The dissonance we experience while looking at this image indicates that we have strong expectations about the basic structural properties of a face that cannot help but influence our perception (see Hancock \& Foster, 2012).

Figure 2: For Gaussian likelihood function $\left(L\left(\theta_{N} \mid \theta\right)\right.$; dashed blue line) and prior distribution $(p(\theta)$; dotted red line) (A) the posterior $\left(p\left(\theta \mid \theta_{N}\right)\right.$; solid black line) is also Gaussian with a mean corresponding to the maximum a posteriori estimate of the attribute value on that trial (B). The maximum a posteriori estimate, $\theta_{\text {MAP }}$, is pulled away from the raw sensory estimate, $\theta_{N}$, towards the peak of the prior, $\theta_{\text {prior }}$ by an amount dependent on the level of uncertainty, $\sigma_{0}$, in the sensory estimate.

Figure 3: (A) Example stimuli as used in Mareschal et al. (2013ab, 2014). Both faces have eyes deviated 9 degrees rightwards but the eyes in the right hand face have noisy texture added and are reduced in contrast. (B) Judgments of gaze deviation using a forced-choice task show a bias to perceive the noisy stimulus as looking more direct (Mareschal et al., 2013b). Bias in the perceived direction of gaze of a test face is plotted as a function of the direction of a comparator. In this experiment either the test or the comparator face had noise added to the eyes (red circles and blue squares, respectively). A signature of a prior for direct gaze is that the slopes of the best fitting lines to these data are oppositely signed depending on whether the increased uncertainty is in the test or the comparator. Here, perceived gaze deviation is "pulled" towards a direction close to (but for this subject slightly rightwards of) zero, indicated by the horizontal gaze deviation at which the lines cross each other and the x-axis. The approximately linear increase in bias magnitude with horizontal gaze deviation is consistent with the prior probability distribution being a roughly Gaussian function of gaze direction (C) Results using a pointer to indicate perceived direction of gaze indicate that the "pull" towards direct gaze is not restricted to horizontal gaze deviations but generalizes across all directions (Mareschal et al., 2014). Here, lines join data points from corresponding stimuli presented in noiseless (solid symbol) and noisy conditions. The resulting needles can be seen to point towards a direction close to direct gaze. It should be noted that the perceptual bias is towards direct gaze per se rather than towards the orientation of the head, as head orientation is 15 degrees leftwards of direct, indicated by the position of the $\mathrm{H}$.

Figure 4: Schematic illustration of how the effects of perceptual adaptation and a prior for direct gaze might both be instantiated within the same channel structure. (A) A stimulus with eyes clearly visible (high certainty) gazing slightly to the right excites channels preferring direct and rightwards gaze and leads to an essentially veridical percept of gaze direction (height of bars indicates level of response of each channel). (B) Adaptation to rightwards averted gaze reduces the sensitivity of the rightwards channel from its unadapted level (dashed line), causing subsequently presented rightwards gaze to appear as more direct (perceptual shift indicated by arrow). (C) The response to a stimulus with poorly visible eyes (low certainty) leads to a reduced response in the direct and rightwards channels such that the baseline response of the leftwards channel is no longer negligible in comparison, causing the perceived direction of gaze to be pulled towards direct. The prior for direct gaze is thus represented implicitly in the central tendency of the channel preferences. 


\section{References:}

Anderson, B.L., O’Vari, J. \& Barth, H. (2011). Non-Bayesian contour synthesis. Current Biology, 21, 492-496.

Armann, R., \& Bülthoff, I. (2012). Male and female faces are only perceived categorically when linked to familiar identities - And when in doubt, he is a male. Vision Research, 63, 69-80.

Calder, A. J., Beaver, J.D., Winston, J.S., Dolan, R.J., Jenkins, R., Eger, E. \& Henson, R.N.A. (2007). Separate coding of different gaze directions in the superior temporal sulcus and inferior parietal lobule, Current Biology, 17(1), 20-25.

Calder, A. J., Jenkins, R., Cassel, A., \& Clifford, C. W. (2008). Visual representation of eye gaze is coded by a non-opponent multichannel system. Journal of Experimental Psychology: General, 137(2), 244-61. doi: 10.1037/0096-3445.137.2.244.

Cellerino, A., Borghetti, D., \& Sartucci, F. (2004). Sex differences in face gender recognition in humans. Brain Research Bulletin, 63, 443-449.

Cheleski, D.J., Mareschel, I., Calder, A. J., \& Clifford, C.W.G. (2013). Eye gaze is not coded by cardinal mechanisms alone, Proceedings of the Royal Society of London, Series B: Biological Sciences, 280, 1764,20131049

Clifford, C.W.G., Webster, M.A., Stanley, G.B., Stocker, A.A., Kohn, A., Sharpee, T.O. \& Schwartz, O. (2007). Visual adaptation: neural, psychological and computational aspects. Vision Research, 47, 3125-3131.

de Waal, F.B.M., \& Pokorny, J.J. (2008). Faces and behinds: Chimpanzee sex perception. Advanced Science Letters, 1, 99-103.

Edwards, M., Badcock, D.R., Smith, A.T. (1998). Independent speed-tuned global-motion systems. Vision Res. 38(11), 1573-1580.

Fischer, B.J., \& Pena, J.L. (2011). Owl's behavior and neural representation predicted by Bayesian inference. Nature Neuroscience, 14(8), 1061-1066.

Fletcher, P.C. \& Frith, C.D. (2009). Perceiving is believing: a Bayesian approach to explaining the positive symptoms of schizophrenia. Nat Rev Neurosci. 10(1), 48-58.

Gaetano, J., van der Zwan, R., Blair, D., \& Brooks, A. (2014). Hands as sex cues: Sensitivity measures, male bias measures, and implications for sex perception mechanisms. PLOS ONE, 9(3): e91032. doi:10.1371/journal.pone.0091032

Ganguli, D., \& Simoncelli, E.P. (2014). Efficient sensory encoding and Bayesian inference with heterogeneous neural populations. Neural Computation, 26(10), 2103-2134. doi: 10.1162/NECO_a_00638

Gerardin, P., Kourtzi, Z., \& Mamassian, P. (2010). Prior knowledge of illumination for 3D perception in the human brain. Proc Natl Acad Sci U S A. 107(37), 16309-16314.

Girshick, A. R., Landy, M. S., \& Simoncelli, E. P. (2011). Cardinal rules: Visual orientation perception reflects knowledge of environmental statistics. Nature Neuroscience, 14 (7), 926-932

Haselton, M. G. \& Buss, D. M. (2000). Error management theory: A new perspective on biases in cross-sex mind reading. Journal of Personality and Social Psychology, 78, 81-91.

Haselton, M. G. \& Nettle, D. (2006). The paranoid optimist: an integrative evolutionary model of cognitive biases. Personality and Social Psychology Review, 10, 47-66.

Hohwy, J., Palmer, C. (2014). Social cognition as causal inference: implications for common knowledge and autism. In Mattia Gallotti and John Michael (Eds.), Social Ontology and Social Cognition, Springer Series "Studies in the Philosophy of Sociality", Vol. 4. 
Jenkins, R., Beaver, J.D., \& Calder, A.J. (2006). I thought you were looking at me: direction-specific aftereffects in gaze perception. Psychological Science, 17 (6). 506-513. doi:10.1111/j.14679280.2006.01736.x

Johnson, K.L., lida, M., \& Tassinary, L.G. (2012). Person (mis)perception: functionally biased sex categorization of bodies. Proceedings of the Royal Society of London, Series B: Biological Sciences, 279(1749), 4982-4989.

Kersten, D., Mamassian, P., \& Yuille, A. (. 2004). Object perception as Bayesian inference. Annu Rev Psychol 55, 271-304.

Kilner JM, Friston KJ, Frith CD. (2007). The mirror-neuron system: a Bayesian perspective. Neuroreport, 18 (6), 619-623.

Knill, D.C. (2003). Mixture models and the probabilistic structure of depth cues. Vision Research, 43(7), 83154.

Knill, D.C. \& Pouget, A. (2004). The Bayesian brain: the role of uncertainty in neural coding and computation. Trends Neurosci. 27(12), 712-719.

Leopold, D. A., O'Toole, A. J., Vetter, T. \& Blanz, V. (2001). Prototype-referenced shape encoding revealed by high-level aftereffects. Nature Neuroscience, 4, 89-94.

Mareschal, I., Calder, A. J., Dadds, M. R., \& Clifford, C. W. (2013a). Gaze categorization under uncertainty: psychophysics and modeling. Journal of Vision, 13(5), 18. doi: 10.1167/13.5.18

Mareschal, I., Calder, A. J., \& Clifford, C. W. G. (2013b). Humans have an expectation that gaze is directed toward them. Current Biology, 23(8), 717-721. doi: 10.1016/j.cub.2013.03.030

Mareschal, I., Otsuka, Y., \& Clifford, C.W. (2014). A generalized tendency toward direct gaze with uncertainty. Journal of Vision, 14(12): 27. doi: 10.1167/14.12.27.

Martin, W.W. \& Jones, R. F. (1982) The accuracy of eye-gaze judgment: a signal detection approach. British Journal of Social Psychology, 21 (4), 293-9.

Martin, W.W. \& Rovira, L.M. (1981). An Experimental analysis of discriminability and bias in eye-gaze judgment. Journal of Nonverbal Behaviour, 5(3), 155-163.

O'Neil, S.F. \& Webster, M.A. (2011). Adaptation and the perception of facial age. Visual cognition, 19(4), 534-550. doi: 10.1080/13506285.2011.561262

Pellicano, E., \& Burr, D. (2012). When the world becomes 'too real': a Bayesian explanation of autistic perception. Trends in Cognitive Sciences, 16(10), 504-10.

Perrett, D. I., Smith, P. A. J., Potter, D. D., Mistlin, A. J., Head, A. S., Milner, A. D., \& Jeeves, M. A. (1985). Visual cells in the temporal cortex sensitive to face view and gaze direction. Proceedings of the Royal Society Series B-Biological Sciences, 223(1232), 293-317. doi: 10.1098/rspb.1985.0003

Rhodes, G., Jeffery, L., Watson, T. L., Clifford, C. W. G., \& Nakayama, K. (2003). Fitting the mind to the world: Face adaptation and attractiveness aftereffects. Psychological Science, 14, 558-566.

Rhodes, G., Jeffery, L., Watson, T. L., Jaquet, E., Winkler, C., Clifford, C. W. G. (2004). Orientation-contingent face aftereffects and implications for face coding mechanisms. Current Biology, 14, 2119-2123.

Rhodes, G., Robbins, R., Jaquet, E., McKone, E., Jeffery, L. \& Clifford, C.W.G. (2005) Adaptation and face perception- how aftereffects implicate norm based coding of faces. In Fitting the mind to the world: adaptation and aftereffects in high-level vision (eds C. W. G. Clifford \& G. Rhodes), Advances in visual cognition series, vol. 2, pp. 213-240. Oxford, UK: Oxford University Press.

Sheldrake, Rupert (2003). The Sense of being stared At, And Other Aspects of the Extended Mind, London: Hutchinson. 
Stocker, A. A., \& Simoncelli, E. P. (2006a). Noise characteristics and prior expectations in human visual speed perception. Nature Neuroscience, 9 (4), 578-585.

Stocker, A. A., \& Simoncelli, E. P. (2006b). Sensory adaptation within a Bayesian framework for perception. In Y. Platt (Eds.). Advance in neural information processing systems (Vol. 18, Weiss, B. \& Schoelkopf, J. pp. 1291-1298). London: Springer.

Troje, N. F., Sadr, J., Geyer, H. \& Nakayama, K. (2006). Adaptation aftereffects in the perception of gender from biological motion. Journal of Vision, 6(8), 850-857.

Trommershäuser, J., Körding, K. P. \& Landy, M. S. (2011). Sensory cue integration. New York, NY: Oxford University Press.

Voelkle, M.C., Ebner, N.C., Lindenberger, U., \& Riediger, M. (2012). Let me guess how old you are: Effects of age, gender, and facial expression on perceptions of age. Psychology and Aging, 27, 265-277.

Wainwright, M.J. (1999). Visual adaptation as optimal information transmission. Vision Research, 39(23), 3960-3974.

Watson, T. (2013), Implications of holistic face processing in autism and schizophrenia. Frontiers in Psychology, 4:414. doi: 10.3389/fpsyg.2013.00414

Watson, T.L. \& Clifford, C.W.G. (2003) Pulling faces: investigating the face distortion aftereffect, Perception, 32, 1109-1116.

Watson, T.L. \& Clifford, C.W.G. (2006) Orientation dependence of the orientation contingent face after effect, Vision Research, 46, 3422-3429.

Webster, M.A. \& MacLeod, D.I. (2011). Visual adaptation and face perception, Philosophical Transactions of the Royal Society B, 366(1571), 1702-1725. doi: 10.1098/rstb.2010.0360

Webster, M.A., Kaping, D., Mizokami, Y., Duhamel, P. (2004). Adaptation to natural facial categories. Nature, 428, 557-561.

Zemel R, Dayan P, Pouget A. (1998). Probabilistic interpretation of population code. Neural Computation, 10(2), 403-430. 